\title{
ANÁLISE ENERGÉTICA DA CULTURA DO CRAMBE (Crambe abyssinica Hochst) PRODUZIDA EM PLANTIO DIRETO ${ }^{1}$
}

\author{
SAMIR P. JASPER ${ }^{2}$, MARCO A. M. BIAGGIONI ${ }^{3}$, PAULO R. A. SILVA ${ }^{4}$, \\ ANDRÉ S. SEKI ${ }^{5}$, OSMAR C. BUENO ${ }^{6}$
}

\begin{abstract}
RESUMO: O objetivo deste trabalho foi caracterizar o consumo de energia na implantação e condução da cultura do Crambe abyssinica Hochst, em sistema de plantio direto, na Fazenda Experimental Lageado, pertencente à Faculdade de Ciências Agronômicas - UNESP, localizada no município de Botucatu - SP. Avaliando a sustentabilidade do sistema, estimaram-se, a eficiência energética e a cultura do crambe. Para o levantamento dos componentes da estrutura de dispêndios, foram utilizados coeficientes energéticos preconizados em literaturas especializadas. As estruturas de dispêndios energéticos por tipo, fonte e forma demonstraram que a energia indireta participou com 65,03\%; as fontes industrial, fóssil e biológica representaram 68,80\%, 23,67\% e 11,30\%, respectivamente. Quanto às formas em que se apresentaram, os fertilizantes químicos contribuíram com 44,31\%, e o óleo diesel, com $23,04 \%$, totalizando $67,35 \%$ do dispêndio energético, evidenciando a dependência do sistema das fontes energéticas industriais e fósseis. A eficiência da cultura encontrada foi de 9,98, apontando que, para cada unidade calórica aplicada na produção do crambe, o retorno foi de 8,98 unidades, a eficiência energética foi superior a 38, demonstrando alta sustentabilidade do sistema agrícola, tendo atingido energia cultural superior a $37 \mathrm{mil} \mathrm{MJ} \mathrm{ha}{ }^{-1}$.
\end{abstract}

PALAVRAS-CHAVE: eficiência energética, matérias-primas para biodiesel, biocombustíveis.

\section{ANALYSIS OF ENERGY IN THE CRAMBE CULTURE (Crambe abyssinica Hochst) PRODUCED IN NO TILLAGE}

\begin{abstract}
The objective of this study was to characterize the energy consumption in the deployment and conduction of the culture of Crambe abysinica Hochst in no-tillage, at the Experimental Farm belonging to the Faculty of Agronomic Sciences - UNESP, located in Botucatu - SP, Brazil. Assessing the sustainability of the system were estimated, energy efficiency and culture of crambe. To survey the components of the structure of expenditures, it was used Energetic coefficients in specialized literature. The structures of energy expenditure by type, source and form, demonstrated that the indirect energy participated with $65.03 \%$, industrial sources, fossil and biological accounted for $68.80 \%, 23.67 \%$ and $11.30 \%$, respectively. The forms have been introduced; chemical fertilizers contributed $44.31 \%$ and diesel to $23.04 \%$ to $67.35 \%$ of energy expenditure, showing the dependence of the system of energy sources and industrial fuels. The efficiency of the culture was found to be 9.98, indicating that for each calorie used in the production of crambe return was 8.98 units, the efficiency was above 38 , demonstrating the high sustainability of the agricultural system to reach energy cultural exceeding 37 thousand $\mathrm{MJ} \mathrm{ha}^{-1}$.
\end{abstract}

KEYWORDS: energetic efficiency, raw materials of biodiesel, biofuels.

\footnotetext{
${ }^{1}$ Extraído da tese de doutorado do primeiro autor.

${ }^{2}$ Eng ${ }^{\mathrm{o}}$ Agrônomo, Pós-Doutorando, Programa de Pós-Graduação em Agricultura, FCA/UNESP, Botucatu - SP, jasper@fca.unesp.br

${ }^{3}$ Eng ${ }^{0}$ Agrônomo, Prof. Doutor, Departamento de Engenharia Rural, FCA/UNESP, Botucatu - SP, biaggioni@ fca.unesp.br

${ }^{4}$ Eng $^{\mathrm{O}}$ Agrônomo, Prof. Doutor, Departamento de Engenharia Rural, FCA/UNESP, Botucatu - SP, arbex@ @ca.unesp.br

${ }^{5}$ Eng ${ }^{\mathrm{O}}$ Agrônomo, Doutorando do Programa de Pós-Graduação em Energia na Agricultura, FCA/UNESP, Botucatu - SP

${ }^{6}$ Eng $^{-}$Agrônomo, Livre Docente em Extensão Rural, Prof. Doutor do Departamento de Gestão e Tecnologia Agroindustrial, FCA/UNESP, Botucatu - SP 


\section{INTRODUÇÃO}

O biodiesel é um biocombustível derivado de biomassa renovável para uso em motores a combustão interna com ignição por compressão ou, conforme regulamento para outro tipo de geração de energia, que possa substituir parcial ou totalmente combustível de origem fóssil (BRASIL, 2005). Assim, há amplas possibilidades de uso do biodiesel em transportes urbanos, rodoviários, ferroviários e aquaviários de passageiros e cargas, geradores de energia, motores estacionários, etc.

De acordo com OLIVEIRA \& COSTA (2005), a implantação de um programa energético com biodiesel abre oportunidades para grandes benefícios sociais decorrentes do alto índice de geração de empregos por capital investido, culminando com a valorização do campo e a promoção do trabalhador rural, além das demandas por mão de obra qualificada para o processamento e, em muitos casos, beneficiamento do óleo vegetal. Salienta-se, entretanto, a reversão no fluxo internacional de capitais, uma vez que o aproveitamento do biodiesel permite uma redução das importações de diesel e a comercialização internacional de Certificados de Redução de Emissões de gases causadores do efeito estufa.

A produção de biodiesel no Brasil encontra-se alicerçada em culturas anuais, principalmente de ciclo primavera/verão, faltando alternativas para o outono/inverno que permitisse dar continuidade à produção de biodiesel, além de realizar rotação de cultura. Para a obtenção de máxima eficiência da capacidade produtiva do solo, o planejamento de rotação de culturas deve considerar, além das espécies comerciais, aquelas destinadas à cobertura do solo, que produzam grandes quantidades de biomassa, cultivadas quer em condição solteira, quer em consórcio com culturas comerciais (EMBRAPA SOJA, 2004).

Atualmente, na produção de biodiesel, empresas e órgãos estaduais e federais vêm priorizando busca por matérias-primas alternativas, contudo sempre avaliando os atributos destas sob aspectos agronômicos e tecnológicos, como: teor de óleo; produtividade; sistema produtivo; ciclo da cultura, etc. Considerando esses aspectos, acredita-se que o Crambe abyssinica Hochst, por tratar-se de cultura de inverno, tem grande potencial para constituir-se em matéria-prima para biodiesel, além de atuar na rotação de cultura. Cultivada em maior escala no México e nos Estados Unidos para produção de óleo industrial, o cultivo iniciou-se no Brasil em 1995, na Fundação MS, no município de Maracaju - MS, porém, na época, a planta era estudada somente para fins de rotação de cultura (ECHEVENGUÁ, 2007).

Estudos realizados na estação de pesquisa da Fundação do Mato Grosso do Sul (2007), em Maracajú - MS, destacaram como vantagens Crambe abyssinica Hochst: tolerância à seca, à geada depois de estabelecida, elevada precocidade e elevado teor de óleo (34\% a 38\%). A produtividade em 2007 variou entre 1.000 e 1.500 quilos por hectare, contudo ressalta-se a possibilidade de aumentar a produtividade.

Dos debates estabelecidos nos últimos anos sobre a questão energética no Brasil, pouca importância se deu ao balanço energético dos sistemas produtivos. Considerações maiores têm sido efetuadas na busca por novas fontes, a partir de culturas com alto potencial de produção calórica. $\mathrm{O}$ conhecimento da relação produção/consumo de energia, tanto quanto a análise econômica, é instrumental básico para elaboração de políticas que definam a quantidade de calorias e proteínas no consumo interno, exportação ou na transformação em combustíveis, permitindo prever o nível de dependência do País nessa área (CARMO et al., 1988; CARMO \& COMITRE, 1991).

O balanço energético visa a estabelecer os fluxos de energia, identificando sua demanda total e eficiência, refletida pelo ganho líquido de energia e pela relação saída/entrada, além da quantidade necessária para produzir ou processar um quilograma de determinado produto. Nesse processo, quantificam-se todos os insumos utilizados e produzidos que são transformados em unidades de energia. A determinação da eficiência energética é importante instrumento no monitoramento da 
sustentabilidade da agricultura ante o uso de fontes de energia não renováveis (BUENO et al., 2000).

Nesse contexto, a eficiência energética pode constituir-se numa interessante ferramenta indicadora de sustentabilidade para analisar a produção do Crambe abyssinica Hochst como fonte de matéria-prima para o biodiesel.

Neste trabalho, teve-se o objetivo de estimar a eficiência energética relativa a produção de Crambe abyssinica Hochst, em sistema de plantio direto.

\section{MATERIAL E MÉTODOS}

O trabalho foi conduzido no ano agrícola de 2008, na Fazenda Experimental Lageado, pertencente à Faculdade de Ciências Agronômicas - UNESP, localizada no município de Botucatu -SP, na região centro-oeste do Estado de São Paulo, tendo como coordenadas geográficas de latitude $22^{\circ} 51^{\prime} \mathrm{S}$ e longitude $48^{\circ} 26^{\prime} \mathrm{W}$ de Greenwich, altitude média de $770 \mathrm{~m}$, declividade média de $4,5 \%$ e clima subtropical chuvoso, apresentando inverno seco, tipo Cfa, de acordo com o critério de Köeppen.

O solo da área experimental foi classificado como Nitossolo Vermelho distroférrico (EMBRAPA, 1979). A área experimental está sendo conduzida no sistema de plantio direto desde 1997 com as culturas do milho ou soja, nas safras de verão, e as culturas da aveia-preta (Avena strigosa Schreb) ou triticale (Triticum turgidocereale), nas safras de outono/inverno.

Após a colheita do milho safrinha de 2008 na área, realizou-se a dessecação por meio de herbicida (Glyfosate $360 \mathrm{~g} \mathrm{~L}^{-1}$ ), na dosagem de $3 \mathrm{~L} \mathrm{ha}^{-1}$, para a instalação do experimento. Foram utilizadas sementes de crambe da variedade brilhante, material este pertencente à Fundação do Mato Grosso do Sul (Fundação MS), com poder germinativo de $85 \%$ e $98 \%$ de pureza. As sementes foram tratadas com $0,3 \mathrm{~L} \mathrm{ha}^{-1}$ de fungicida (Thiram $200 \mathrm{~g} \mathrm{~kg}^{-1}$ ). Na adubação de base, utilizou-se de $200 \mathrm{~kg} \mathrm{ha}^{-1}$ do fertilizante 08-28-16.

A área experimental utilizada foi de um hectare, sendo distribuídas nesta área quatro parcelas de $200 \mathrm{~m}^{2}$, com $20 \mathrm{~m}$ de comprimento e $10 \mathrm{~m}$ de largura.

Na operação de semeadura, utilizou-se um trator da marca John Deere, modelo $6600(4 \times 2$ TDA), com potência nominal de $89 \mathrm{~kW}(121 \mathrm{cv})$. Para as operações de pulverização e transporte do crambe, foi utilizado o trator da marca Massey Ferguson, modelo MF 283 (4x2 TDA), com potência nominal de $63,2 \mathrm{~kW}(86 \mathrm{cv})$.

Os implementos utilizados para a instalação e semeadura do crambe foram: pulverizador de barras marca Jacto, modelo Condor 600 e semeadora-adubadora de precisão, da marca Semeato, modelo SHM 15/17, de arrasto, acionado por controle remoto com 15 linhas, espaçados em 0,17 m.

Na colheita do crambe, foram utilizados os seguintes equipamentos: colhedora autopropelida de grãos, marca Massey Ferguson, modelo MF 3640, tração traseira auxiliar, com potência nominal de $95,6 \mathrm{~kW}(130 \mathrm{cv})$ e uma carreta basculante marca Stara, modelo Reboke 5000.

Os parâmetros avaliados foram: velocidade de deslocamento e consumo horário de combustível. Para a aquisição de dados, utilizou-se de um sistema de aquisição digital "micrologger 21X, marca Campbell Scientific", para monitorar e exibir os dados do gerador de impulso (fluxômetro). Os dados foram armazenados continuamente, em um módulo de armazenamento externo de dados "Storage module SM196". O monitoramento da velocidade foi realizado indiretamente por meio da frequência de aquisição de dados de $10 \mathrm{~Hz}$ do "micrologger $21 \mathrm{X}$ ".

Para quantificar o consumo de combustível, utilizou-se de um fluxômetro, marca "Flowmate" oval, modelo Oval M-III, o qual emitia ao sistema de aquisição de dados uma unidade de pulso a cada $\mathrm{mL}$ de combustível que passava pelo mesmo. O consumo operacional de combustível foi determinado por meio da relação entre o consumo horário de combustível e a capacidade de campo 
efetiva. A capacidade de campo efetiva foi determinada pela relação entre a área útil da parcela trabalhada e o tempo gasto no percurso da parcela.

\section{Análise energética}

A confecção da matriz energética iniciou-se pela definição do agrossistema, caracterizando-se o período enfocado, detalhando-se, em seguida, o itinerário técnico percorrido, sendo elaboradas, a seguir, as chamadas rubricas operacionais. Os itens que compuseram a matriz de exigência física da cultura foram transformados em coeficientes energéticos. O sistema foi delimitado de forma a englobar as atividades relativas à dessecação, semeadura e colheita do crambe, incluindo todos os processos e gastos energéticos embutidos na atividade. O desempenho do sistema foi dado pela eficiência cultural, que apresenta a relação entre Saídas Úteis e Entradas Culturais, e pela eficiência energética, que, segundo RISOUD (1999), representa a relação entre o somatório das energias brutas dos produtos / somatório das energias não renováveis.

Como energia direta do sistema, foi considerada energia biológica (mão de obra e sementes) e energia fóssil (óleo diesel, lubrificante e graxa), diretamente consumido no sistema agrícola (BONNY, 1993). Como energia indireta, considerou-se a energia industrial, empregada na fabricação de equipamentos (máquinas e implementos) e insumos (fertilizantes e defensivos) e outros "inputs" necessários à produção (CLEVELAND, 1995). Para o cálculo, foi utilizada metodologia específica desenvolvida por DOERING III et al. (1977), para quantificar os "inputs" de energia indireta utilizadas no sistema delimitado, por meio da depreciação energética, segundo os dias de utilização e em função da vida útil dos equipamentos.

\section{Coeficientes energéticos/conversão energética}

Mão de obra: Com relação ao cálculo da energia investida pelos trabalhadores rurais nas diferentes operações do itinerário técnico, seguiu-se a metodologia proposta por CARVALHO et al. (1974) e descritas em BUENO (2002). Seguindo a metodologia de CARVALHO et al. (1974), procedeu-se a determinação do GER (Gasto Energético no Repouso) de cada trabalhador. As equações determinam o gasto energético no repouso em kcal, e o dispêndio calórico final diário é apresentado em MJ.

Insumos: Os valores energéticos dos elementos foram obtidos por meio da multiplicação da massa (kg) ou volume (L) pelo conteúdo energético específico correspondente, apresentado pela bibliografia ponderando o nível de utilização por hectare na cultura.

Fertilizantes: Os valores adotados para os elementos são os informados por BUENO (2002): $\mathrm{N} 62,61 \mathrm{MJ} \mathrm{kg}^{-1} ; \mathrm{P}_{2} \mathrm{O}_{5} 9,63 \mathrm{MJ} \mathrm{kg}^{-1} ; \mathrm{K}_{2} \mathrm{O} 9,21 \mathrm{MJ} \mathrm{kg}^{-1}$, com acréscimo de 0,50 MJ em 63,45\% do fertilizante químico devido ao transporte marítimo da importação (ANDA, 2009). Multiplicaram-se as quantidades efetivas dos elementos ativos ( $\mathrm{N}, \mathrm{P} 2 \mathrm{O} 5$ e $\mathrm{K} 2 \mathrm{O}$, em $\mathrm{kg}$ ) pelo valor energético correspondente.

Fungicidas e Herbicidas: os coeficientes energéticos são informados por PIMENTEL (1980), considerando, 271,71 e 631,83 MJ por kg de ingrediente ativo, necessária para a formulação do fungicida e do herbicida, respectivamente.

Máquinas e equipamentos: Utilizou-se da metodologia desenvolvida por DOERING III et al. (1977), empregada por diversos autores (SERRA et al., 1979; ULBANERE, 1988; BEBER, 1989; COMITRE, 1993; CAMPOS et al., 2004; entre outros), baseada na depreciação energética que, a partir da massa das máquinas, consiste em depreciá-las durante sua vida útil. Os coeficientes adotados foram (COMITRE, 1993): máquinas 14.628,68 $\mathrm{MJ} \mathrm{t}^{-1}$; colhedora 13.012,57 $\mathrm{MJ} \mathrm{t}^{-1}$; implemento (até semeadura) 8.628,99 $\mathrm{MJ} \mathrm{t}^{-1}$ e implemento (pós-semeadura) 8.352,67 $\mathrm{MJ} \mathrm{t}^{-1}$. 1993).

Pneus: considerou-se o coeficiente energético para os pneus de 85.829,40 $\mathrm{MJ} \mathrm{t}^{-1}$ (COMITRE,

Combustíveis e lubrificantes: Óleo diesel, óleo lubrificante e graxa, com poderes caloríficos de 40,88 $\mathrm{MJ} \mathrm{L}^{-1}, 37,75 \mathrm{MJ} \mathrm{L}^{-1}$ e 43,38 $\mathrm{MJ} \mathrm{kg}^{-1}$, respectivamente (ASAE, 1997). 
Crambe: 27,40 MJ kg-1 (Laboratório de Recursos Naturais - FCA/UNESP - ABNT-NBR 8633).

\section{RESULTADOS E DISCUSSÃO}

A produtividade do Crambe abyssinica Hochst foi de 1.507,05 $\mathrm{kg} \mathrm{ha}^{-1}$, acima do potencial produtivo de $1.400 \mathrm{~kg} \mathrm{ha}^{-1}$, informado no catálogo da Fundação MS, detentora da cultivar brilhante, utilizada no experimento, com 33,98\% de teor de óleo, resultando numa produtividade superior a $500 \mathrm{~L} \mathrm{ha}^{-1}$.

Na Tabela 1, apresentam-se as entradas de energia, na forma direta e indireta e a eficiência, considerando-se os valores médios utilizados para cálculo de produção anual para um hectare da cultura.

TABELA 1. Estrutura de dispêndios, por tipo, fonte e forma; "entradas" culturais, "saídas" úteis, energia cultural líquida e eficiência cultural da produção de crambe, por hectare.

Structure of expenditures, by type, source and form, "inputs" cultural, "outpus" useful, energy cultural and efficiency of the culture the crambe production per hectare.

\begin{tabular}{lr}
\hline Tipo, Fonte e Forma & Entradas Culturais (MJ) \\
\hline Energia Direta & $1.447,57$ \\
\hline Biológica & \\
Mão de obra & 0,67 \\
$\quad$ Tratorista & 1,18 \\
$\quad$ Braçal & 465,84 \\
$\quad$ Semente & \\
Fóssil $\quad 953,73$ \\
Óleo Diesel & 12,85 \\
Lubrificante & 13,30 \\
Graxa & $2.691,93$ \\
\hline Energia Indireta & 30,70 \\
\hline Industrial & 827,07 \\
Máquinas e implementos & $1.000,16$ \\
Herbicidas e fungicidas & 539,28 \\
Nitrogênio & 294,72 \\
Fósforo & $4.139,50$ \\
Potássio & $41.293,17$ \\
\hline "Entradas” culturais (A) & $37.153,67$ \\
"Saídas” úteis (B) & 38,96 \\
\hline Energia Cultural Líquida (B - A) & 9,98 \\
\hline Eficiência Energética & \\
\hline Eficiência Cultural (B $\div$ A) & \\
\hline
\end{tabular}

O maior consumo de energia no sistema em estudo correspondeu ao fertilizante, na forma de nitrogênio, com 24,16\% do total (Figura 1). Em seguida, ficou o óleo diesel com 23,04\%, fato explicado pela utilização de equipamentos, como tratores, nas fases de dessecação e semeadura, e colhedora automotriz. Dados os problemas relativos ao custo de energia e à esgotabilidade, e considerando a crise energética que é atualmente vivenciada, o elevado consumo de energia na forma de combustível torna o item o principal "estrangulador" do presente sistema em estudo. As soluções visando à redução de consumo energético dentro dessa rubrica são complicadas, uma vez que estariam todas vinculadas à necessidade de redução de utilização desses equipamentos. 


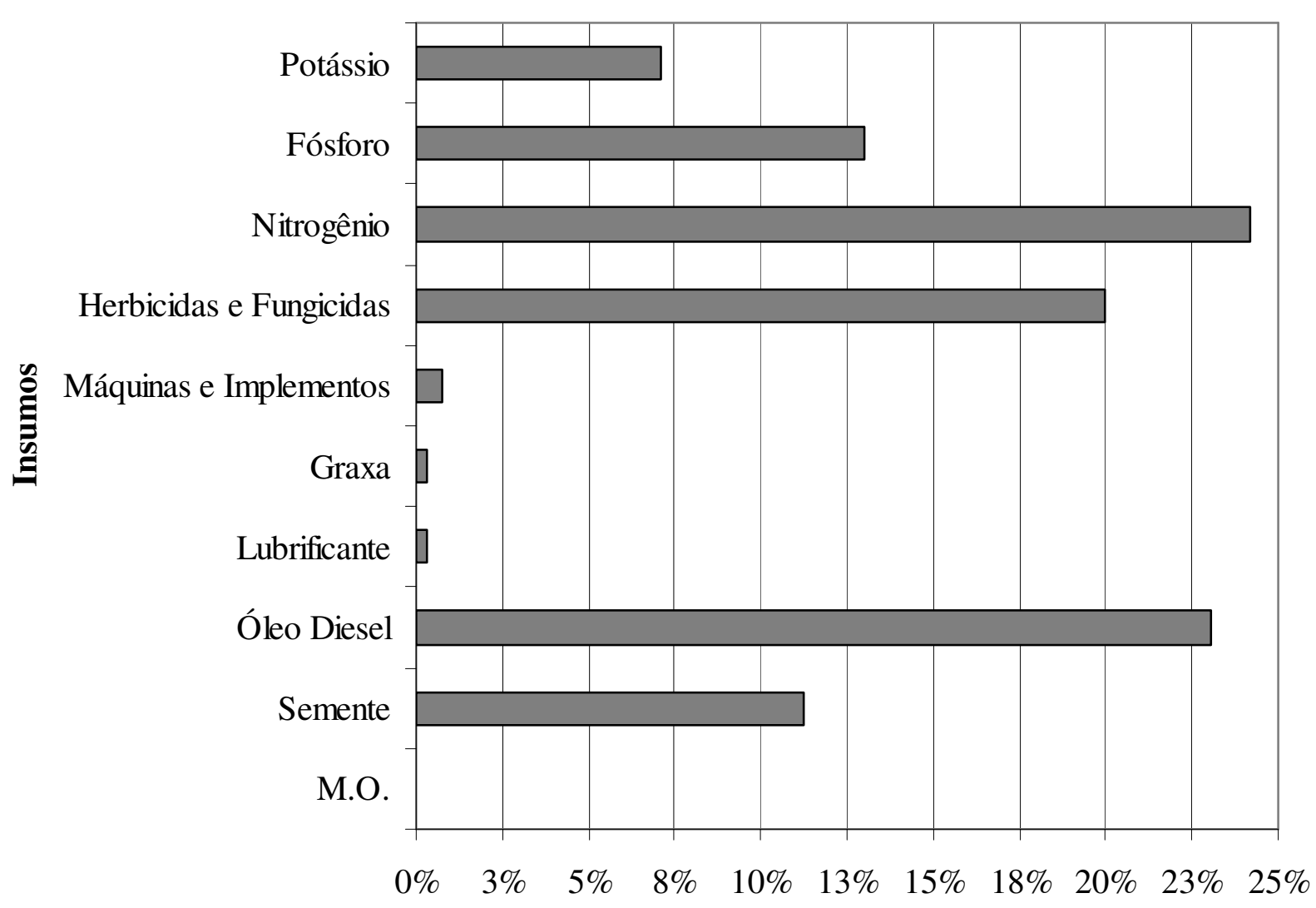

Participação no consumo de energia

FIGURA 1. Participação percentual dos diversos insumos ("inputs") na formação da eficiência energética Crambe abyssinica Hochst. Percentage of various inputs on the formation of efficiency energy Crambe abyssinica Hochst.

De toda a energia gasta na adubação (1.834,16 $\left.\mathrm{MJ} \mathrm{ha}^{-1}\right), 54,53 \%$ foram empregados em nitrogênio, que possui elevado coeficiente energético, o que onera o consumo. $\mathrm{O}$ fósforo teve participação no consumo energético muito menor que a do nitrogênio e até do potássio.

As proporções utilizadas de energia direta $(34,97 \%)$ e indireta $(65,03 \%)$ mantiveram-se em torno de $1 / 3$ e $2 / 3$, respectivamente, em relação ao total da energia consumida pelo sistema. A produção do crambe analisada dependeu, fundamentalmente, de fontes de energia industriais, particularmente fertilizantes químicos $(44,31 \%)$ e de fontes fósseis $(23,04 \%)$, apresentando tendência semelhante aos trabalhos de BEBER (1989) e ULBANERE (1988).

A participação energética do combustível (Figura 1), nessa análise, foi inferior aos dados constantes na bibliografia para outras culturas, de forma geral. QUESADA et al. (1987) informam participações de 47,6\%; 46,5\% e 40,7\% na energia advinda do óleo diesel, para as culturas de milho, soja e trigo, respectivamente, em estudo desenvolvido no Rio Grande do Sul, fato explicado devido ao número reduzido de operações mecanizadas da cultura do crambe quando comparada com essas culturas.

Em todas as etapas, houve baixo consumo de energia na forma de trabalho humano. Isso motiva autores, como ULBANERE (1988), a desconsiderar essa rubrica na contabilização dos consumos de energia. Neste trabalho, os valores baixos ocorreram devido às características da delimitação do sistema em estudo, que considera somente a mão de obra para as atividades mecanizadas. 


\section{Eficiência energética}

Analisando a Tabela 1, verifica-se que a energia cultural líquida para produção do crambe foi de 37.153,67 $\mathrm{MJ} \mathrm{ha}^{-1}$, resultando numa eficiência da cultura de 9,98, ou seja, a cada unidade de energia empregada na produção de crambe, o retorno é de 8,98 unidades de energia. A eficiência energética foi de 38,96, representado que, para cada unidade utilizada de energia fóssil, o retorno é de 37,96 unidades de energia na produção de crambe. MELO et al. (2007) encontraram, para a cultura da soja, uma energia cultural líquida de $67.641,11 \mathrm{MJ} \mathrm{ha}^{-1}$, porém a entrada de energia foi 12.502,36 $\mathrm{MJ} \mathrm{ha}^{-1}$, três vezes superior à encontrada do crambe, de 4.139,50 $\mathrm{MJ} \mathrm{ha}^{-1}$, o que contribui para uma eficiência da cultura $84,47 \%$ inferior ao do crambe.

SATO et al. (2008) ao analisarem energeticamente a cultura do pinhão-manso, relataram eficiência da cultura de 0,25 , além de ressaltar que este sistema não depende de fontes não renováveis, como óleo diesel, logo não sendo possível calcular a eficiência energética.

Apesar das intensas atividades com máquinas agrícolas tratorizadas e intensiva utilização de insumos energéticos, principalmente de derivados de petróleo, notadamente fertilizantes, a eficiência energética para a produção do Crambe abyssinica Hochst mostrou-se bastante favorável. Em termos comparativos, de forma teórica, SCHROLL (1994), por exemplo, sugere que um índice de 2 para a relação saída/entrada de energia ("output"/"input") seria razoável para uma política de desenvolvimento da sustentabilidade ecológica da Dinamarca.

\section{CONCLUSÕES}

Análise energética é favorável para produção de crambe de cada unidade empregada de energia, tendo-se o retorno de 8,98 unidades, além da elevada energia da cultura líquida.

Analisando globalmente o sistema de produção do crambe, o maior consumo energético ocorreu, principalmente, na forma de fertilizantes, energia de fonte industrial, seguido pelo óleo diesel, energia de fonte fóssil.

Somando o dispêndio energético com fertilizantes, defensivos e combustíveis fósseis, totalizaram-se $88,70 \%$ do consumo, mostrando alta dependência dessas energias, tornando evidente a busca de soluções alternativas para o sistema de produção do crambe.

\section{AGRADECIMENTOS}

Ao CNPq, pela concessão de bolsa de doutorado.

\section{REFERÊNCIAS} ABNT. ASSOCIAÇÃO BRASILEIRA DE NORMAS TÉCNICAS. NBR 8633: Determinação do
poder calorífico superior. Rio de Janeiro, 1984. 13 p.

ANDA. ASSOCIAÇÃO NACIONAL PARA DIFUSÃO DE ADUBOS. Estatísticas: produção nacional de fertilizantes intermediários e importação de fertilizantes intermediários. São Paulo, 2009. Disponível em: <www.anda.org.br/estatisticas.aspx>. Acesso em: 15 abr. 2009.

ASAE. D497.3. Agricultural Machinery Management Data. ASAE Standarts (I) 1996. St Joseph, 1997. p. 364-370.

BEBER, J.A.C. Eficiência energética e processos de produção em pequenas propriedades rurais, Agudo - RS. 1989. 295 f. Dissertação (Mestrado em Extensão Rural) - Universidade Federal de Santa Maria, Santa Maria, 1989.

BONNY, S. Is agriculture using more and more energy ? A French case study. Agricultural Systems, Kidlington, v.43, n.1, p.51-66, 1993. 
BRASIL. Lei no 11.097 , de 13 de janeiro de 2005. Dispõe sobre a criação do Programa Nacional de Produção e Uso do Biodiesel e sobre a adição de biodiesel ao óleo diesel. Disponível em: $<$ http://www.anp.gov.br>. Acesso em: 15 maio 2009.

BUENO, O. C. Análise energética e eficiência cultural do milho em assentamento rural. 2002. 146 f. Tese (Doutorado em Energia na Agricultura) - Faculdade de Ciências Agronômicas, Universidade Estadual Paulista, Botucatu, 2002.

BUENO, O.C.; CAMPOS, A.T.; CAMPOS, A.T. Balanço de energia e contabilização da radiação global: simulação e comparativo. In: AVANCES en Ingeniería Agrícola. Buenos Aires: Editorial Facultad de Agronomia, 2000. p.477-482.

CAMPOS, A.T.; SAGLIETTI, J.R.C.; BUENO, O.C.; CAMPOS, A.T.; KLOSOWSKI, E.S.; GASPARINO, E. Balanço energético na produção de feno de alfafa em sistema intensivo de produção de leite. Ciência Rural, Santa Maria, v.34, n.1, p.245-251, 2004.

CARMO, M.S.; COMITRE, V. Evolução do balanço energético nas culturas de soja e milho no Estado de São Paulo. In: CONGRESSO BRASILEIRO DE ECONOMIA E SOCIOLOGIA RURAL, 29., 1991, Campinas. Anais... Brasília: SBESR, 1991. p.131-149.

CARMO, M.S.; COMITRE, V.; DULLEY, R.D. Balanço energético de sistemas de produção na agricultura alternativa. Agricultura em São Paulo, São Paulo, v.35, n.1, p.87-97, 1988.

CARVALHO, A.; GONÇALVES, G.G.; RIBEIRO, J.J.C. Necessidades energéticas de trabalhadores rurais e agricultores na sub-região vitícola de "Torres". Oeiras: Instituto Gulbenkian de Ciência, Centro de Estudos de Economia Agrária, 1974. 79 p.

CLEVELAND, C.J. The direct and indirect use of fossil fuels and electricity in USA agriculture, 1910-1990. Agriculture, Ecosystems and Environment, Amsterdam, v.55, n.2, p.111-121, 1995.

COMITRE, V. Avaliação energética e aspectos econômicos da filière soja na região de Ribeirão Preto - SP. 1993. 152 f. Dissertação (Mestrado em Planejamento Agropecuário) - Universidade Estadual de Campinas, Campinas, 1993.

DOERING III, O.C.; CONSIDINE, T.J.; HARLING, C.E. Accouting for tillage equipment and othermachinery in agricultural energy analysis. West Lafayette: Purdue University, 1977. 4 p. (Agr. Exp. Sta. NSF/RA - 770128).

ECHEVENGUÁ, A. Crambe surge como nova opção para produzir biodiesel. 2007. Disponível em: <www.ecoeacao.com.br>. Acesso em: 9 maio 2009.

EMBRAPA. EMPRESA BRASILEIRA DE PESQUISA AGROPECUÁRIA. Manual de métodos de análise de solo. Rio de Janeiro: Serviço Nacional de Levantamento e Conservação dos Solos, 1979. $227 \mathrm{p}$.

EMBRAPA SOJA. EMPRESA BRASILEIRA DE PESQUISA AGROPECUÁRIA. Tecnologias de Produção da Soja: Rotação de Culturas. Londrina, 2004. Disponível em:

<www.cnpso.embrapa.br/producaosojaPR/rotacao.htm>. Acesso em: 15 maio 2009.

FUNDAÇÃO DO MATO GROSSO DO SUL. Culturas para biodiesel, Crambe: Maracajú, 2007. Disponível em: <www.fundacaoms.com.br>. Acesso em: 15 maio 2009.

MELO, D.; PEREIRA, J.O.; SOUZA, E.G.; FILHO, A.G.; NÓBREGA, L.H.P.; NETO, R.P. Balanço energético do sistema de produção de soja e milho em uma propriedade agrícola do oeste do Paraná. Acta Scientiarum Agronomy, Maringá, v.29, n.2, p.173-178, 2007.

OLIVEIRA, L.B.; COSTA, A.O. da. Biodiesel: uma experiência de desenvolvimento sustentável. 2005. Disponível em: <http://www.biodieselecooleo.com.br/biodiesel/estudos>. Acesso em: 25 maio 2009. 
PIMENTEL, D. Energy inputs for the production formulation, packaging, and transport for various pesticides. In: PIMENTEL, D. (Ed.). Handbook of energy utilization in agriculture. Boca Raton: CRC Press, 1980. p.45-48.

QUESADA, G.M.; BEBER, J.A.C.; SOUZA, S.P. de. Balanços energéticos: uma proposta metodológica para o Rio Grande do Sul. Ciência e Cultura, São Paulo, v.39, n.1, p.20-28, 1987.

RISOUD, B. Développement durable et analyse énergétique d'exploitations agricoles. Économie Rurale, Paris, n.252, p.16-27, juillet-août, 1999.

SATO, M.; BUENO, O.C.; ESPERANCINI, M.S.T. Análise energética da cultura do pinhão-manso (Jatropha curcas L.) em sistema de sequeiro: estudo de caso. Energia na Agricultura, Botucatu, v.23, n.1, p.90-98, 2008.

SCHROLL, H. Energy-flow and ecological sustainability in Danish agriculture. Agriculture, Ecosystems and Environment, Amsterdam, v.51, n.3, p.301-310, 1994.

SERRA, G.; HEEZEN, A.M.; MOREIRA, J.R.; GOLDEMBERG, J. Avaliação da energia investida na fase agrícola de algumas culturas. Brasília: Secretaria de Tecnologia Industrial, 1979. $86 \mathrm{p}$.

ULBANERE, R.C. Análise dos balanços energético e econômico relativa à produção e perda de grãos de milho no Estado de São Paulo. 1988. 127 f. Tese (Doutorado em Energia na Agricultura) Faculdade de Ciências Agronômicas, Universidade Estadual Paulista, Botucatu, 1988. 